\title{
Extreme nonlinear optics of two-level systems
}

\author{
T. Tritschler, O. D. Mücke, and M. Wegener \\ Institut für Angewandte Physik, Universität Karlsruhe (TH), Wolfgang-Gaede-Straße 1, 76131 Karlsruhe, Germany
}

(Received 24 June 2003; published 12 September 2003)

\begin{abstract}
For Rabi frequencies comparable to, or even larger than, the transition frequency of a two-level system, the regime of extreme nonlinear optics is reached. Here, we give an overview of the radiated light intensity as a function of carrier frequency of light, transition frequency, Rabi frequency, spectrometer frequency, as well as of the shape and duration of the exciting optical pulses. The graphical representations reveal an amazing complexity and beauty of the nonlinear optical response. Analytical results within the "square-wave approximation" qualitatively reproduce many of the intricate features of the exact numerical calculations.
\end{abstract}

DOI: 10.1103/PhysRevA.68.033404

PACS number(s): 42.50.Md, 42.50.Hz, 42.65.Ky

\section{INTRODUCTION}

For many systems such as solids, molecules, or atoms, the light-matter interaction can be described by a two-level system to first approximation. Although the two-level system is one of the most fundamental paradigms of both quantum mechanics and nonlinear optics [1-3], its behavior for excitation with extremely intense femtosecond optical pulses still reveals surprises.

The intensity of light is best encoded in the Rabi frequency $\Omega_{R}$, which is proportional to the laser electric field. The regime of traditional nonlinear optics, i.e., $\Omega_{\mathrm{R}} / \Omega \ll 1$ with the transition frequency $\Omega$ has been studied extensively. The regime of extreme nonlinear optics, on the other hand, where $\Omega_{\mathrm{R}} / \Omega \approx 1$ or even $\Omega_{\mathrm{R}} / \Omega \gg 1$, has attracted much less attention. This might have to do with the fact that this regime was considered inaccessible, unphysical, or irrelevant for a long time. Indeed, in the famous textbook of Allen and Eberly [1] the authors write on page 42: “... Obviously it becomes questionable whether the existence of an atom is possible in the presence of such an external field. Certainly, resonant transitions could not be defined. The consequence is that we may safely take the inequality (in words Rabi frequency $\ll$ transition frequency) to be well-satisfied in every situation of interest in optical resonance."

Recent experiments on semiconductors with $\Omega / \omega_{0} \approx 1$ $[4,5]$ and $\Omega / \omega_{0} \approx 2[6]$, where $\omega_{0}$ is the carrier frequency of the exciting laser pulses, have shown that this is no longer true. There, the regime of $\Omega_{\mathrm{R}} / \omega_{0} \approx 1$ was indeed reached at laser intensities in excess of $10^{12} \mathrm{~W} / \mathrm{cm}^{2}$, and a description in terms of two-level systems has been able to reproduce the experimental results amazingly well. Thus, it is natural to ask what would happen for yet larger Rabi frequencies. A few important publications can be found in Refs. [7-11]. However, we are not aware of any work which gives a complete overview of the rich behavior as a function of the four involved frequencies: Carrier frequency of light $\omega_{0}$, transition frequency $\Omega$, Rabi frequency $\Omega_{\mathrm{R}}$, and spectrometer frequency $\omega$. It is the aim of this work to give such an overview. Only from the corresponding graphical representations the amazing beauty of the nonlinear optical response becomes apparent. One of the interesting aspects is that peaks can occur at the spectral position of even harmonics, even though the two-level system has inversion symmetry. While such effects have recently been discussed on two different experimental examples [4-6], it turns out that they also occur at many other points within the complete parameter space.

This paper is organized as follows: In Sec. II we briefly define the equations of motion under investigation. After presenting a rather complete overview on the basis of exact numerical calculations in Sec. III, we discuss the "frozenwave approximation" in Sec. IV and the "square-wave approximation" in Sec. V before concluding in Sec. VI.

\section{DEFINITION OF THE PROBLEM}

Within the dipole approximation [3], but without employing the rotating wave approximation and without transverse or longitudinal damping [12], the Bloch equations of a twolevel system with transition frequency $\Omega$ for the Bloch vector $(u, v, w)^{\mathrm{T}}$ can be written in matrix form as

$$
\left(\begin{array}{c}
\dot{u} \\
\dot{v} \\
\dot{w}
\end{array}\right)=\left(\begin{array}{ccc}
0 & +\Omega & 0 \\
-\Omega & 0 & -2 \Omega_{\mathrm{R}}(t) \\
0 & +2 \Omega_{\mathrm{R}}(t) & 0
\end{array}\right)\left(\begin{array}{c}
u \\
v \\
w
\end{array}\right) .
$$

The dots denote the derivative with respect to time $t$. Here we have introduced the (instantaneous) Rabi frequency $\Omega_{\mathrm{R}}(t)$ with

$$
\hbar \Omega_{\mathrm{R}}(t)=d E(t)
$$

with the dipole matrix element $d$ and the laser electric field

$$
E(t)=\widetilde{E}(t) \cos \left(\omega_{0} t+\phi\right) .
$$

$\widetilde{E}(t)$ is the electric-field envelope, $\omega_{0}$ the carrier frequency of light, and $\phi$ the so-called carrier-envelope phase. Note that the Rabi frequency itself oscillates with the carrier frequency of light and periodically changes sign. We shall call the peak of the Rabi frequency $\Omega_{\mathrm{R}}$ [rather than $\Omega_{\mathrm{R}}(t)$ ] with $\hbar \Omega_{\mathrm{R}}=d \widetilde{E}_{0}$, where $\widetilde{E}_{0}$ is the peak of the electric-field envelope. As usual, $w(t)$ is the inversion of the two-level system. The macroscopic optical polarization entering into the Maxwell equations is given by the dipole density of two-level systems times the (real) component $u(t)$ of the Bloch vector. 


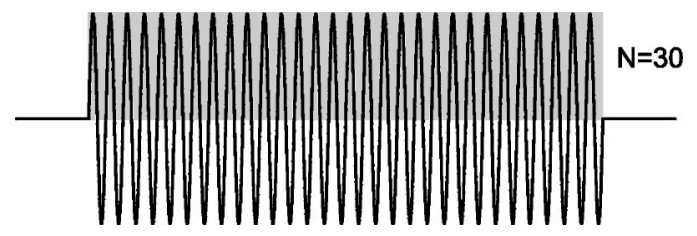

FIG. 1. Illustration of the box-shaped optical pulses $E(t)$ used in Figs. 2 and 3. The integer number of cycles in the pulse is called $N$. The gray area indicates the electric-field envelope $\widetilde{E}(t)$.

The light intensity radiated by the two-level system is proportional to the square modulus of the second temporal derivative of the polarization, hence proportional to $\left|\omega^{2} u(\omega)\right|^{2}$ in the Fourier domain, where $\omega$ is the spectrometer frequency. It is natural to relate all frequencies to the carrier frequency of light $\omega_{0}$, in which case the dependence of the radiated intensity on the three dimensionless parameters $\Omega / \omega_{0}, \Omega_{\mathrm{R}} / \omega_{0}$, and $\omega / \omega_{0}$ has to be studied. In all calculations, we start from the ground state of the two-level system, i.e., from Bloch vector $(0,0,-1)^{\mathrm{T}}$.
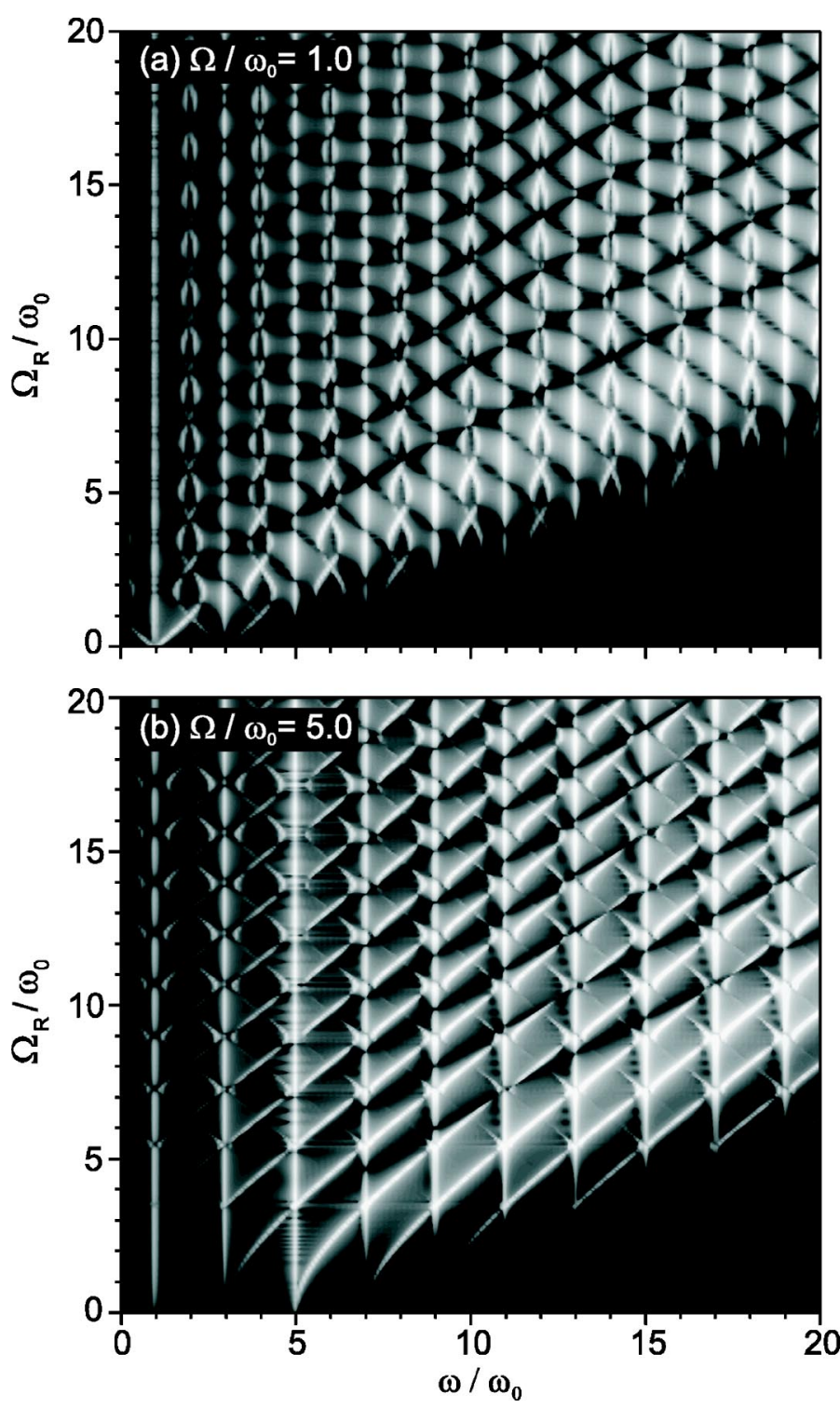

\section{OVERVIEW USING EXACT NUMERICAL CALCULATIONS}

The simplest and cleanest situation is given when the envelope Rabi frequency is either constant or zero. Let us start our discussion with such box-shaped optical pulses (illustrated in Fig. 1), which are $N=30$ optical cycles in duration (for, e.g., $\hbar \omega_{0}=1.5 \mathrm{eV}$, this would roughly correspond to a $90 \mathrm{fs}$ long pulse). To get an overview we can either fix $\Omega / \omega_{0}$ and depict the radiated intensity versus $\Omega_{\mathrm{R}} / \omega_{0}$ and $\omega / \omega_{0}$ (Fig. 2) or, alternatively, fix $\Omega_{\mathrm{R}} / \omega_{0}$ and plot the signals versus $\Omega / \omega_{0}$ and $\omega / \omega_{0}$ (Fig. 3).

For $\Omega_{\mathrm{R}} / \omega_{0} \ll 1$ on the vertical axis of Fig. 2(a), where $\Omega / \omega_{0}=1$ (resonant excitation), conventional Rabi oscillations [13] occur and the well-known Mollow triplet [14] can be seen at $\omega / \omega_{0}=1$ on the horizontal axis. At larger $\Omega_{\mathrm{R}} / \omega_{0}$ approaching unity, carrier-wave Rabi oscillations $[15,16]$ take place and additional carrier-wave Mollow triplets [4] appear around odd integers $\omega / \omega_{0}$. Beyond $\Omega_{\mathrm{R}} / \omega_{0}=1$, the Mollow sidebands are "repelled" by the central peaks of the adjacent Mollow triplets. They oscillate around even integer

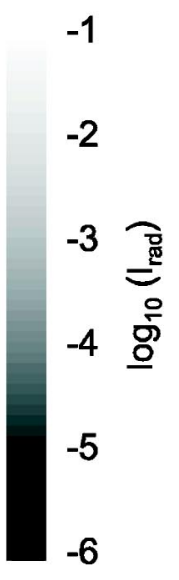

FIG. 2. Gray-scale images of the radiated intensity spectra $I_{\text {rad }}(\omega)$ $\propto\left|\omega^{2} u(\omega)\right|^{2} \quad$ (normalized and on a logarithmic scale) from exact numerical solutions of the two-level system Bloch equations (1). The peak Rabi frequency $\Omega_{\mathrm{R}}$ of the exciting $N=30$ cycles long box-shaped optical pulses, illustrated in Fig. 1, is plotted along the vertical axis. The transition frequency $\Omega$ is parameter. (a) $\Omega / \omega_{0}=1$ and (b) $\Omega / \omega_{0}=5$. $\omega_{0}$ is the carrier frequency of the laser pulses. 

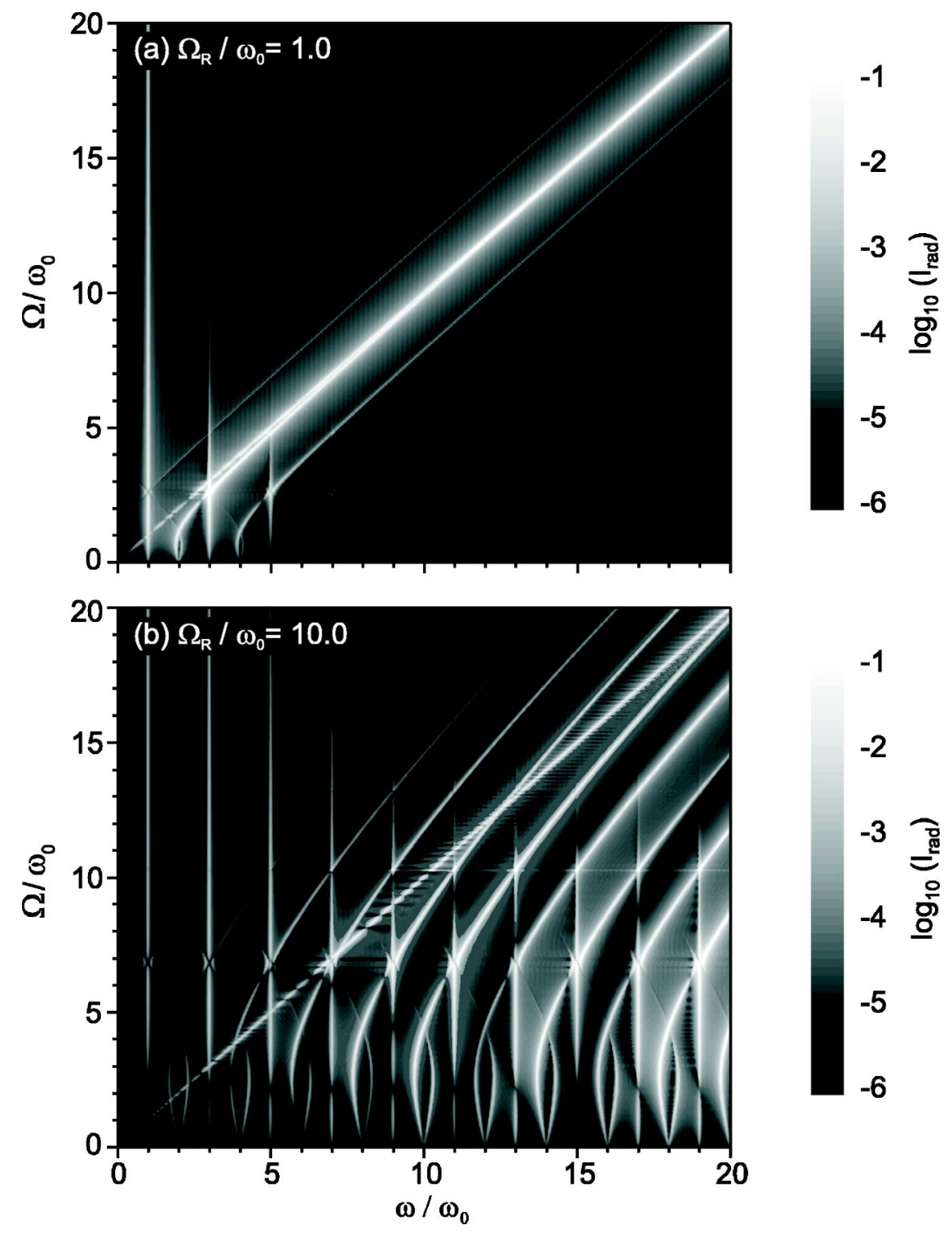

FIG. 3. Same as Fig. 2, but versus transition frequency $\Omega$ for two fixed values of the peak Rabi frequency $\Omega_{\mathrm{R}}$. (a) $\Omega_{\mathrm{R}} / \omega_{0}=1$ and (b) $\Omega_{\mathrm{R}} / \omega_{0}=10$.

values of $\omega / \omega_{0}$ and finally converge towards these values in the limit $\Omega_{\mathrm{R}} / \omega_{0} \gg 1$. On the way, they periodically cross at even integers $\omega / \omega_{0}$ versus $\Omega_{\mathrm{R}} / \omega_{0}$ with a period of $\pi / 2$ for $\Omega_{\mathrm{R}} / \omega_{0} \gg 1$ (whereas the first crossing occurs at $\Omega_{\mathrm{R}} / \omega_{0} \approx 1$ [4]). For off-resonant excitation, e.g., $\Omega / \omega_{0}=5$ in Fig. 2(b), the behavior is different for $\Omega_{\mathrm{R}} / \omega_{0}<1$ and $\Omega_{\mathrm{R}} / \omega_{0} \approx 1$, but becomes similar to Fig. 2(a) for $\Omega_{\mathrm{R}} / \omega_{0} \gg 1$.

The other way to look at the parameter space is to fix the Rabi frequency $\Omega_{R} / \omega_{0}$. For large $\Omega / \omega_{0}$ but not too large peak Rabi frequencies $\Omega_{R} / \omega_{0}$ in Fig. 3, well separated high harmonics are observed, as expected from traditional nonlinear optics. On the diagonal, where $\omega=\Omega$, very large resonant enhancement effects are observed. This is also true for the adjacent harmonics at spectrometer frequencies $\omega=\Omega$ $\pm 2 M \omega_{0}$ with integer $M$, which altogether leads to a band of enhancement around the diagonal in Fig. 3. Especially note that large contributions can occur at the spectral positions of even harmonics, as already discussed for Fig. 2. These contributions are especially pronounced for even integer values of $\Omega / \omega_{0}[6]$.
For more realistic smoothed box-shaped optical pulses the overall behavior remains the same. If, e.g., the electric-field envelope is switched on and off by Fermi functions rising and decaying within a few optical cycles, respectively, the behavior of Fig. 3 is slightly smeared out and the contributions at spectrometer frequencies $\omega=\Omega \pm 2 M \omega_{0}$ decay more rapidly for large values of $\Omega / \omega_{0}$ as compared to box-shaped optical pulses.

For other pulse envelopes, the envelope Rabi frequency is not constant within the pulse, which effectively averages along the vertical axis of Fig. 2. This is further illustrated in Fig. 4 for the example of Gaussian pulses with an electricfield envelope given by $\widetilde{E}(t)=\widetilde{E}_{0} \exp \left(-\left(t / t_{0}\right)^{2}\right)$. The temporal full width at half maximum (FWHM) of the intensity profile is given by $t_{\mathrm{FWHM}}=t_{0} 2 \sqrt{\ln \sqrt{2}}$ and translates into a FWHM of $N=t_{\mathrm{FWHM}} /\left(2 \pi / \omega_{0}\right)$ optical cycles in Fig. 4. In Fig. 4(a), where $N=30$ and $\phi=0$, the anticipated averaging can be seen clearly. As a result, the contributions at odd integers $\omega / \omega_{0}$ have almost disappeared in favor of even con- 

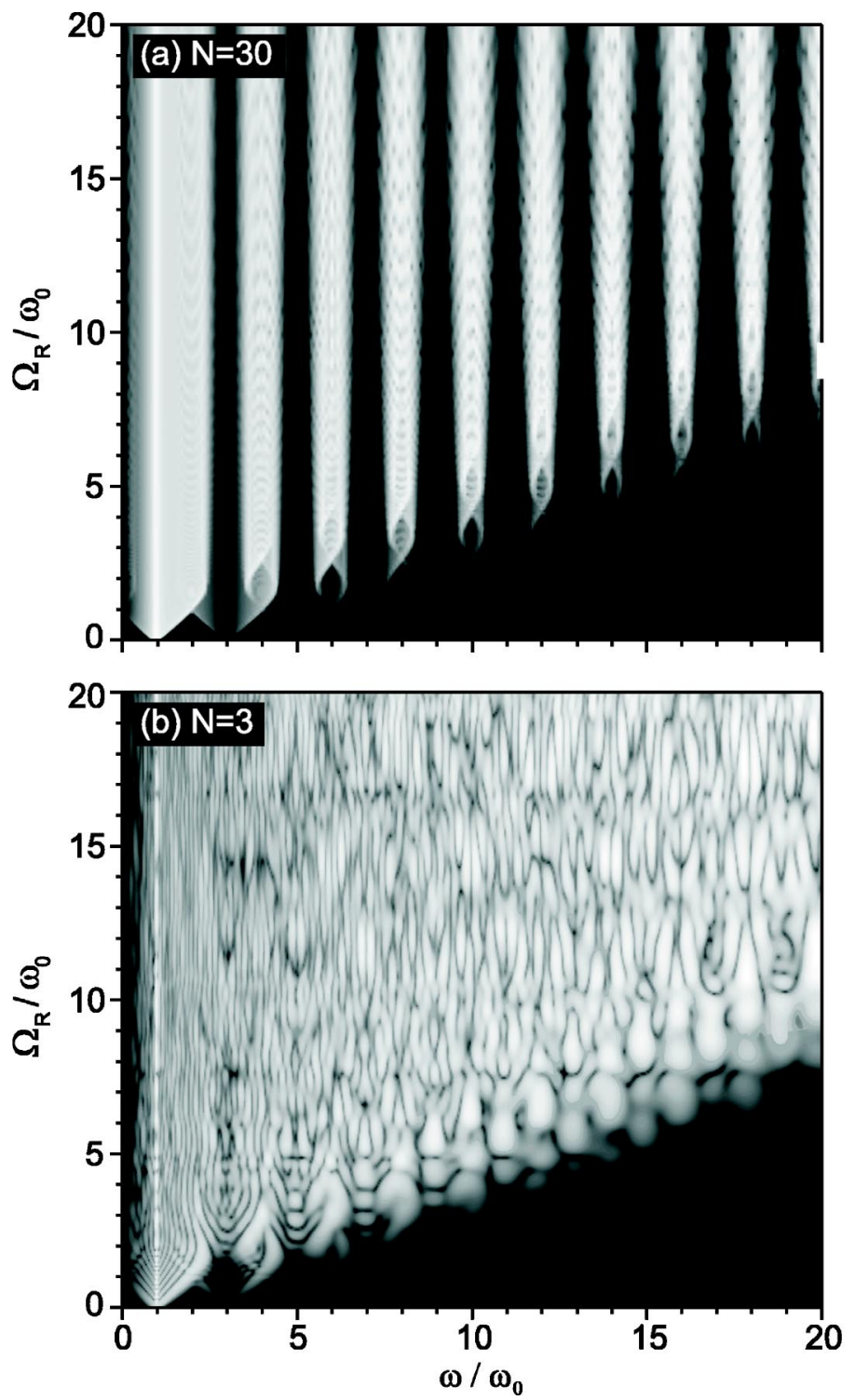

FIG. 4. Same as Fig. 2(a), i.e., $\Omega / \omega_{0}=1$, but for Gaussian optical pulses with $\phi=0$ and with a FWHM of (a) $N=30$ and (b) $N=3$ optical cycles. tribution. This is exactly opposite to what one might have expected intuitively. For $N=30$ and $\phi=\pi / 2$ (not shown), the behavior is similar, apart from fine details which are hardly visible on this scale. For few-cycle optical pulses [Fig. 4(b)] the various contributions get largely broadened spectrally and their mutual interference leads to rather "messy" spectra, which have lost all of the beautiful fine details of Fig. 2. It is clear that this interference also introduces a dependence on the carrier-envelope phase $\phi$ as discussed in detail in Refs. [4-6].

One might be tempted to argue that the peaks at even integers $\omega / \omega_{0}$ in the optical spectra at large $\Omega_{R} / \omega_{0}$ arise from the fact that the complete system, i.e., two-level system plus light field, no longer has inversion symmetry at large electric fields. This reasoning is, however, not consistent with the Bloch equations. Space inversion means that we have to replace $\vec{r} \rightarrow-\vec{r}$. As a result, the dipole matrix element transforms as $d \rightarrow-d$ and the electric field as $E(t) \rightarrow$ $-E(t)$. Hence, we have $\hbar^{-1} d E(t)=\Omega_{\mathrm{R}}(t) \rightarrow+\Omega_{\mathrm{R}}(t)$ and the Bloch equations (1) remain completely unchanged under space inversion. Thus, the solution for the Bloch vector $(u(t), v(t), w(t))^{\mathrm{T}}$ is also unchanged. Finally, the macroscopic optical polarization, which is given by $P(t)$ $=n_{2 \mathrm{LS}} d u(t)$ with the concentration of two-level systems $n_{2 \mathrm{LS}}$, transforms according to $P(t) \rightarrow-P(t)$. Consequently, in an expansion of the polarization in terms of powers of the electric field up to infinite order, strictly no even orders occur, even for arbitrarily large electric fields. Thus, we deliberately avoid to call a peak at, e.g., $\omega / \omega_{0}=2$, secondharmonic generation (SHG). As discussed in detail in Ref. [6], a strict definition of SHG must be based on its carrier frequency or its phase, which are $2 \omega_{0}$ and $2 \phi$, respectively. The peaks at $\omega / \omega_{0}=2$ from the two-level system are not consistent with this definition. Thus, in Ref. [6] we have introduced the notion of third-harmonic generation in disguise of second-harmonic generation for this unusual contribution. A similar argument obviously holds for all the other even integers $\omega / \omega_{0}$, which must not be called even harmonics in the framework of nonlinear optics. In most of the literature-written before the importance of the carrier- 
envelope phase $\phi$ had been appreciated-these peaks are nevertheless called even harmonics.

\section{FROZEN-WAVE APPROXIMATION}

For times much shorter than a cycle of light, $2 \pi / \omega_{0}$, we can employ the frozen-wave approximation, i.e., we approximate $\Omega_{\mathrm{R}}(t)=\Omega_{\mathrm{R}}$ as constant in time. In this limit, it is straightforward to solve the Bloch equations (1) analytically.
This leads to the Bloch vector

$$
\left(\begin{array}{l}
u(t) \\
v(t) \\
w(t)
\end{array}\right)=\mathcal{M}(t)\left(\begin{array}{c}
u(0) \\
v(0) \\
w(0)
\end{array}\right),
$$

with the $(3 \times 3)$ rotation matrix

$$
\mathcal{M}(t)=\left(\begin{array}{ccc}
\frac{4 \Omega_{\mathrm{R}}^{2}+\Omega^{2} \cos \left(\Omega_{\mathrm{eff}} t\right)}{\Omega_{\mathrm{eff}}^{2}} & \frac{\Omega}{\Omega_{\mathrm{eff}}} \sin \left(\Omega_{\mathrm{eff}} t\right) & \frac{2 \Omega \Omega_{\mathrm{R}}}{\Omega_{\mathrm{eff}}^{2}}\left(\cos \left(\Omega_{\mathrm{eff}} t\right)-1\right) \\
-\frac{\Omega}{\Omega_{\mathrm{eff}}} \sin \left(\Omega_{\mathrm{eff}} t\right) & \cos \left(\Omega_{\mathrm{eff}} t\right) & -\frac{2 \Omega_{\mathrm{R}}}{\Omega_{\mathrm{eff}}} \sin \left(\Omega_{\mathrm{eff}} t\right) \\
\frac{2 \Omega \Omega_{\mathrm{R}}}{\Omega_{\mathrm{eff}}^{2}}\left(\cos \left(\Omega_{\mathrm{eff}} t\right)-1\right) & \frac{2 \Omega_{\mathrm{R}}}{\Omega_{\mathrm{eff}}} \sin \left(\Omega_{\mathrm{eff}} t\right) & \frac{\Omega^{2}+4 \Omega_{\mathrm{R}}^{2} \cos \left(\Omega_{\mathrm{eff}} t\right)}{\Omega_{\mathrm{eff}}^{2}}
\end{array}\right) .
$$

Obviously, all three components of the Bloch vector oscillate with the effective frequency $\Omega_{\text {eff }}$, which is given by

$$
\Omega_{\mathrm{eff}}=\sqrt{4 \Omega_{\mathrm{R}}^{2}+\Omega^{2}}
$$

Remember that this frozen-wave approximation is only justified for times $t \ll 2 \pi / \omega_{0}$, hence relevant in the limit $\Omega_{\text {eff }} / \omega_{0} \gg 1$. It can be viewed as the opposite of the rotating wave approximation [1-3]. There, almost nothing is supposed to happen on the time scale of light, whereas here all the significant dynamics takes place within an optical cycle. For $\Omega_{\mathrm{R}} / \Omega \gg 1$, we have $\Omega_{\text {eff }} \approx 2 \Omega_{\mathrm{R}}$, which means that twice the peak Rabi frequency is the largest occurring frequency, hence the highest harmonic generated is roughly given by $\omega / \omega_{0} \approx 2 \Omega_{\mathrm{R}} / \omega_{0}$. This has previously been discussed in Ref. [8].

Starting from the ground state, i.e., from Bloch vector $(0,0,-1)^{\mathrm{T}}$ at time $t=0$, the inversion according to Eqs. (4) and (5) is given by

$$
w(t)=-\frac{\Omega^{2}+4 \Omega_{\mathrm{R}}^{2} \cos \left(\Omega_{\mathrm{eff}} t\right)}{\Omega_{\mathrm{eff}}^{2}} .
$$

Thus, the two-level system can even perform Rabi oscillations for far off-resonant conditions, i.e., for $\Omega / \omega_{0} \gg 1$, if the intensity is so large that it roughly corresponds to a Rabi frequency of $\Omega_{\mathrm{R}} / \Omega=1$, which leads to $w(t)=-1 / 5$ $-4 / 5 \cos \left(\sqrt{5} \Omega_{\mathrm{R}} t\right)$ with maximum inversion $w=+3 / 5$ ( $\Leftrightarrow 80 \%$ maximum occupation of the excited state). It is clear that, within a quantum optical description of the light field, this behavior could be interpreted as multiphoton carrier-wave Rabi oscillations.

\section{SQUARE-WAVE APPROXIMATION}

The Bloch equations (1) describe rotations of the Bloch vector on the Bloch sphere. Within the regime of extreme nonlinear optics, the behavior becomes "enriched" by the fact that one of the rotation frequencies, namely, $2 \Omega_{\mathrm{R}}(t)$ itself oscillates with the carrier frequency of light and periodically changes sign. This oscillation is sinusoidal, yet one might ask whether it is really so important that it is sinusoidal. Having in mind what we have said about the frozenwave approximation in Sec. IV, it is simple to extend that result to piecewise constant electric fields $E(t)$ or Rabi frequencies $\Omega_{\mathrm{R}}(t)$, respectively. This leads us to investigating the square-wave approximation in which we approximate the Rabi frequency for constant envelope via

$$
\Omega_{\mathrm{R}}(t)=\Omega_{\mathrm{R}} \cos \left(\omega_{0} t+\phi\right) \rightarrow \frac{2}{\pi} \Omega_{\mathrm{R}} \operatorname{sgn}\left(\cos \left(\omega_{0} t+\phi\right)\right),
$$

where the signum function is defined as $\operatorname{sgn}(x)=+1$ for $x$ $>0, \operatorname{sgn}(x)=-1$ for $x<0$, and $\operatorname{sgn}(x)=0$ for $x=0$. The prefactor $2 / \pi$ ensures that the average Rabi frequency within half an optical cycle is the same for the square-wave approximation and the exact problem. In that half of the optical cycle where the Rabi frequency is positive (negative), the Bloch vector rotates via the matrix $\mathcal{M}_{+}\left(\mathcal{M}_{-}\right)$, where $\mathcal{M}_{ \pm}$ results from $\mathcal{M}$ by replacing $\Omega_{\mathrm{R}} \rightarrow \pm(2 / \pi) \Omega_{\mathrm{R}}$ in Eqs. (5) and (6). For more than half an optical cycle, the dynamics of the Bloch vector is described by

$$
\left(\begin{array}{c}
u(t) \\
v(t) \\
w(t)
\end{array}\right)=\mathcal{M}_{\mathrm{tot}}(t)\left(\begin{array}{c}
u(0) \\
v(0) \\
w(0)
\end{array}\right),
$$



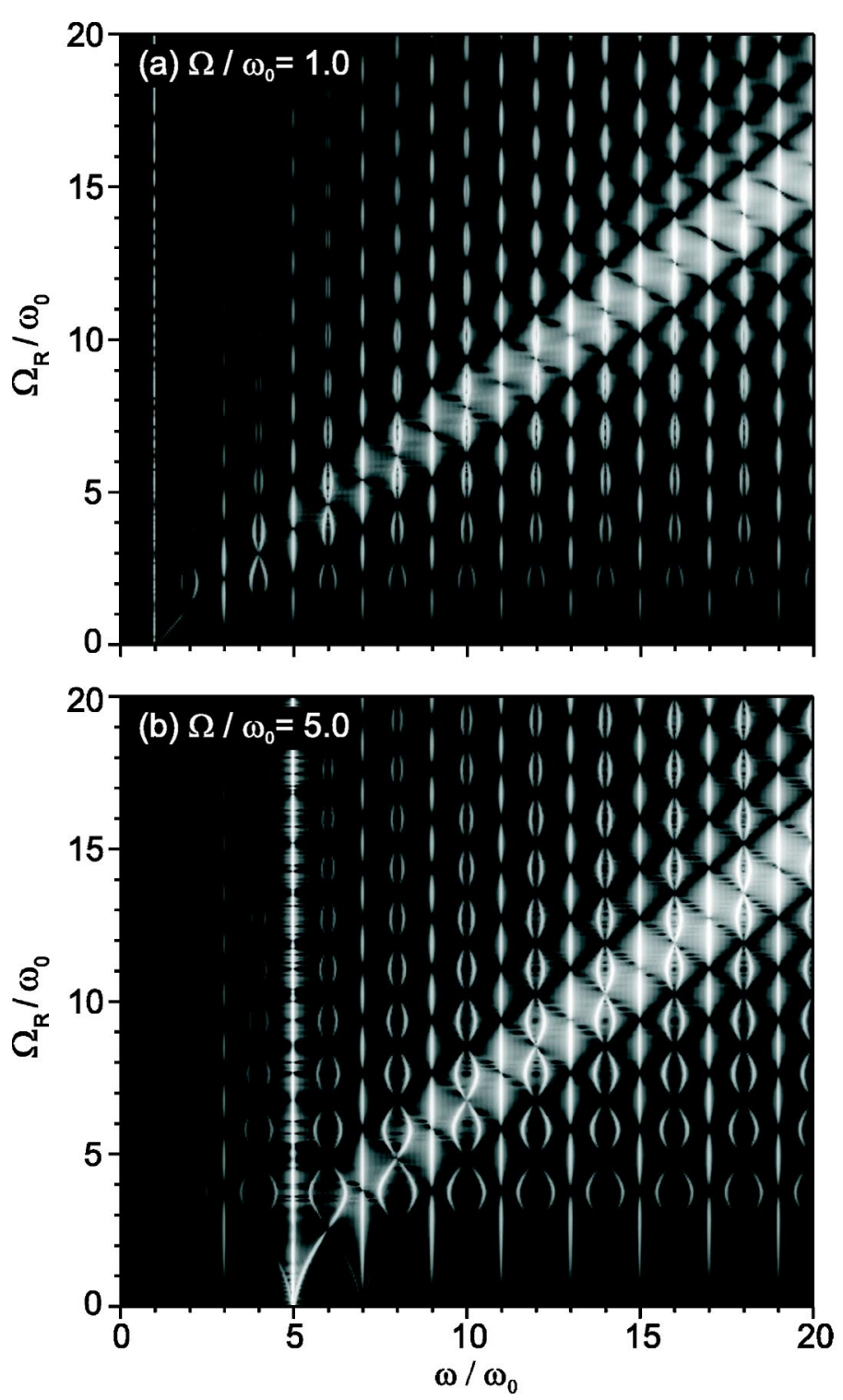

where the total matrix $\mathcal{M}_{\text {tot }}$ is a product of simple analytical $(3 \times 3)$ rotation matrices: For times $t$ after the $N$-cycle long pulse we have

$$
\mathcal{M}_{\mathrm{tot}}(t)=\mathcal{M}_{0}\left(t-N \frac{2 \pi}{\omega_{0}}\right)\left[\mathcal{M}_{-}\left(\frac{\pi}{\omega_{0}}\right) \mathcal{M}_{+}\left(\frac{\pi}{\omega_{0}}\right)\right]^{N}
$$

where $\mathcal{M}_{0}$ results from $\mathcal{M}$ by replacing $\Omega_{\mathrm{R}} \rightarrow 0$ in Eqs. (5) and (6). Within the optical pulse, we get for times $t$ with $\Omega_{\mathrm{R}}(t)>0$

$$
\mathcal{M}_{\mathrm{tot}}(t)=\mathcal{M}_{+}\left(t-N_{t} \frac{2 \pi}{\omega_{0}}\right)\left[\mathcal{M}_{-}\left(\frac{\pi}{\omega_{0}}\right) \mathcal{M}_{+}\left(\frac{\pi}{\omega_{0}}\right)\right]^{N_{t}}
$$

and for times $t$ with $\Omega_{\mathrm{R}}(t)<0$,

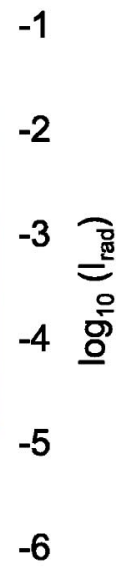

FIG. 5. Same as Fig. 2, but based on the analytical solution of the two-level system Bloch equations within the square-wave approximation. (a) $\Omega / \omega_{0}$ $=1$ and (b) $\Omega / \omega_{0}=5$.

$$
\begin{aligned}
\mathcal{M}_{\text {tot }}(t)= & \mathcal{M}_{-}\left[t-\left(N_{t}+\frac{1}{2}\right) \frac{2 \pi}{\omega_{0}}\right] \mathcal{M}_{+}\left(\frac{\pi}{\omega_{0}}\right) \\
& \times\left[\mathcal{M}_{-}\left(\frac{\pi}{\omega_{0}}\right) \mathcal{M}_{+}\left(\frac{\pi}{\omega_{0}}\right)\right]^{N_{t}} .
\end{aligned}
$$

Here we have introduced the integer number of cycles $N_{t}$ completed up to time $t$ :

$$
N_{t}=\operatorname{int}\left(\frac{\omega_{0} t}{2 \pi}\right)
$$

The value of the integer function (also known as the "Gauss bracket") $\operatorname{int}(x)$ is given by the largest integer $\leqslant x$.

We first test the square-wave approximation by depicting its solutions in Fig. 5. Parameters are identical to those of the exact numerical calculations in Fig. 2. The overall qualitative agreement is amazing, especially for Figs. 2(a) and 5(a). There, $\Omega / \omega_{0}=1$ (resonant excitation), which is nothing but the generalization of Rabi oscillations and Mollow triplets. 
For instance, the periodically occurring constrictions of the repelling Mollow sidebands at even integers $\omega / \omega_{0}$ versus $\Omega_{\mathrm{R}} / \omega_{0}$ with period $\pi / 2$ (see discussion in Sec. III) are very nicely reproduced. For off-resonant excitation $\left(\Omega / \omega_{0}=5\right)$ in Figs. 2(b) and 5(b), the square-wave approximation is less convincing. This aspect can be understood intuitively. For resonant excitation $\left(\Omega / \omega_{0}=1\right)$ the transition frequency resonantly enhances those frequency components of the square wave which have frequency $\omega_{0}$. Thus, the artificial higher harmonics of the square wave at frequencies $3 \omega_{0}, 5 \omega_{0}, \ldots$ are relatively suppressed. Clearly, the square-wave approximation does not properly recover the limit of linear optics, in the sense that $u(t)$ is not sinusoidal in that limit (as it should be), equivalent to higher harmonics of the carrier frequency $\omega_{0}$ in the Fourier domain. Thus, the lower right-hand side of Figs. 5(a) and 5(b) [which is dark in Figs. 2(a) and 2(b)] is an obvious artifact of the square-wave approximation. This artifact is unimportant because we are rather interested in the regime of extreme nonlinear optics.

The simplest cases of commensurability of the frequencies $\omega_{0}, \Omega$, and $\Omega_{\mathrm{R}}$ within the square-wave approximation are given by

$$
\Omega_{\mathrm{eff}} \frac{\pi}{\omega_{0}}=M 2 \pi,
$$

with integer $M$, for which we have

$$
\mathcal{M}_{+}\left(\frac{\pi}{\omega_{0}}\right)=\mathcal{M}_{-}\left(\frac{\pi}{\omega_{0}}\right)=\left(\begin{array}{lll}
1 & 0 & 0 \\
0 & 1 & 0 \\
0 & 0 & 1
\end{array}\right) \text {. }
$$

Under these conditions, an integer number of Rabi oscillations is completed after half an optical cycle. Inserting

$$
\Omega_{\mathrm{eff}}=\sqrt{4\left(\frac{2}{\pi} \Omega_{\mathrm{R}}\right)^{2}+\Omega^{2}}
$$

into Eq. (14), we get that commensurability occurs for specific Rabi frequencies according to

$$
\frac{\Omega_{\mathrm{R}}}{\omega_{0}}=\frac{\pi}{2} \sqrt{M^{2}-\frac{1}{4}\left(\frac{\Omega}{\omega_{0}}\right)^{2}}
$$

with $M=1,2,3, \ldots$ For these Rabi frequencies, peaks at even integers

$$
\frac{\omega}{\omega_{0}}=\frac{\Omega_{\text {eff }}}{\omega_{0}}=2 M
$$

are observed in the optical spectrum, apart from the less interesting peaks at odd integers $\omega / \omega_{0}$, which also occur in traditional nonlinear optics. These peaks at even integers $\omega / \omega_{0}$ form the sloped bright band in Fig. 5, whereas the other even integers $\omega / \omega_{0}$ are absent in the spectrum. This band is less pronounced in the exact numerical solutions (Fig. 2). There, in contrast to the square-wave approxima- tion, the instantaneous Rabi frequency $\Omega_{\mathrm{R}}(t)$ varies within half an optical cycle (somewhat similar to a "chirped" optical pulse), which also introduces peaks at other even integers $\omega / \omega_{0}$. This altogether shows that the constrictions formed by the crossing Mollow triplets in Fig. 2 can be interpreted as points of commensurability of the carrier frequency of light $\omega_{0}$, the transition frequency $\Omega$, and the peak Rabi frequency $\Omega_{R}$. Here an integer number of Rabi oscillations is completed after half an optical cycle and, thus, peaks at even integers $\omega / \omega_{0}$ occur in the optical spectrum. For, e.g., $M$ $=1$ and $\Omega / \omega_{0}=1$ in Eq. (17), we get $\Omega_{\mathrm{R}} / \omega_{0}=\sqrt{3} \pi / 4$ $\approx 1.36$ (Fig. 5), which roughly agrees with $\Omega_{\mathrm{R}} / \omega_{0} \approx 1$ in the exact numerical calculations (Fig. 2). For integers $M$ $\gg \Omega / \omega_{0}$ we get $\Omega_{\mathrm{R}} / \omega_{0}=M \pi / 2$. This period of $\pi / 2$ is also precisely found in the exact numerical calculations (Fig. 2). It is also implicitly contained in Eq. (15) of Ref. [8], which holds under certain approximations specified there and arises from the zeros of the Bessel function $J_{0}$. For large Rabi frequencies, commensurability is easily achieved and these "even harmonics" become the rule rather than the exception, despite the fact that the two-level system has inversion symmetry. In between these points of commensurability, it takes some optical cycles to again approach the initial state. In the Fourier domain, this obviously corresponds to nearby sidebands around those even integers $\omega / \omega_{0}$ (see Figs. 2 and 5).

\section{CONCLUSIONS}

We have given a systematic overview of the nonlinear optical response of the two-level system in the regime of extreme nonlinear optics as a function of carrier frequency of light $\omega_{0}$, transition frequency $\Omega$, peak Rabi frequency $\Omega_{\mathrm{R}}$, spectrometer frequency $\omega$, and optical pulse shape and duration. A part of the intricate fine structures can be understood within the square-wave approximation. Within that approximation, exact analytical results are derived.

It remains to be seen which of the described aspects can be observed in actual experiments in future. Let us recall, however, that the regime of $\Omega_{\mathrm{R}} / \omega_{0}=1$ has recently been subject of several experimental studies in solids, for which the two-level system has provided an adequate description indeed. It thus seems conceivable to us that an increase of the Rabi frequency towards $\Omega_{\mathrm{R}} / \omega_{0}=2$ or more is possible. It is more than likely that interesting deviations from the twolevel system response will be found in any specific system with more than just two electronic levels, especially if continua of states or correlation effects are involved. Nevertheless, the simple two-level system-one of the most fundamental paradigms of nonlinear optics-can still serve as a reference point.

\section{ACKNOWLEDGMENTS}

The work of M.W. was supported by Project Nos. DFG-We 1497/11-1 and DFG-We 1497/9-1. We thank U. Morgner and F. X. Kärtner for stimulating discussions. 
[1] L. Allen and J.H. Eberly, Optical Resonance and Two-Level Atoms (Dover Publications, New York, 1987).

[2] P. Meystre and M. Sargent III, Elements of Quantum Optics, 2nd ed. (Springer, New York, 1991).

[3] W. Schäfer and M. Wegener, Semiconductor Optics And Transport Phenomena, Advanced Texts in Physics (Springer, New York, 2002).

[4] O.D. Mücke, T. Tritschler, M. Wegener, U. Morgner, and F.X. Kärtner, Phys. Rev. Lett. 89, 127401 (2002).

[5] O.D. Mücke, T. Tritschler, M. Wegener, U. Morgner, F.X. Kärtner, G. Khitrova, and H.M. Gibbs (unpublished).

[6] T. Tritschler, O.D. Mücke, M. Wegener, U. Morgner, and F.X. Kärtner, Phys. Rev. Lett. 90, 217404 (2003).

[7] R. Bavli and H. Metiu, Phys. Rev. Lett. 69, 1986 (1992).

[8] M.Yu. Ivanov, P.B. Corkum, and P. Dietrich, Laser Phys. 3, 375 (1993).

[9] A. Levinson, M. Segev, G. Almogy, and A. Yariv, Phys. Rev. A
49, R661 (1994).

[10] T. Zuo, S. Chelkowski, and A.D. Bandrauk, Phys. Rev. A 49, 3943 (1994).

[11] S. Hughes, Phys. Rev. A 62, 055401 (2000).

[12] Damping is irrelevant under the conditions of this paper unless the damping rate becomes comparable to the transition frequency, in which case no resonance exists at all. For our numerical calculations, we chose a transverse relaxation time $T_{2}$, which would, e.g., correspond to $T_{2}=50 \mathrm{fs}$ for a carrier frequency of light $\omega_{0}$ with light period $2 \pi / \omega_{0}=2.8$ fs or $\hbar \omega_{0}$ $=1.5 \mathrm{eV}$, equivalent to about $800 \mathrm{~nm}$ wavelength of light. Longitudinal damping is neglected.

[13] I.I. Rabi, Phys. Rev. 51, 652 (1937).

[14] B.R. Mollow, Phys. Rev. 188, 1969 (1969).

[15] S. Hughes, Phys. Rev. Lett. 81, 3363 (1998).

[16] O.D. Mücke, T. Tritschler, M. Wegener, U. Morgner, and F.X. Kärtner, Phys. Rev. Lett. 87, 057401 (2001). 